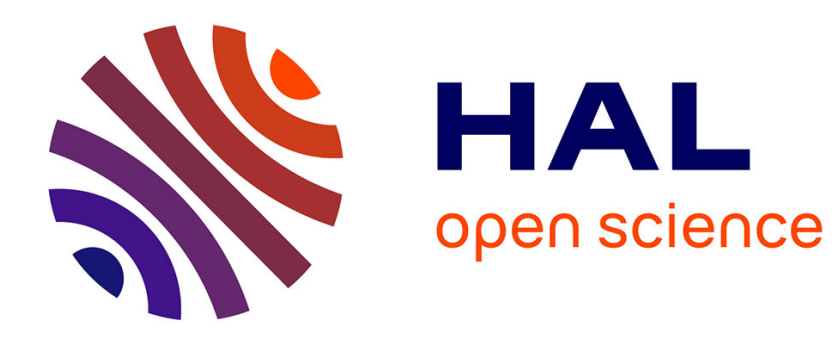

\title{
A Simple Probabilistic Approach of the Yard-Sale Model Christophe Chorro
}

\section{To cite this version:}

Christophe Chorro. A Simple Probabilistic Approach of the Yard-Sale Model. 2015. halshs-01222500

\section{HAL Id: halshs-01222500 \\ https://shs.hal.science/halshs-01222500}

Submitted on 30 Oct 2015

HAL is a multi-disciplinary open access archive for the deposit and dissemination of scientific research documents, whether they are published or not. The documents may come from teaching and research institutions in France or abroad, or from public or private research centers.
L'archive ouverte pluridisciplinaire HAL, est destinée au dépôt et à la diffusion de documents scientifiques de niveau recherche, publiés ou non, émanant des établissements d'enseignement et de recherche français ou étrangers, des laboratoires publics ou privés. 


\section{Documents de Travail du

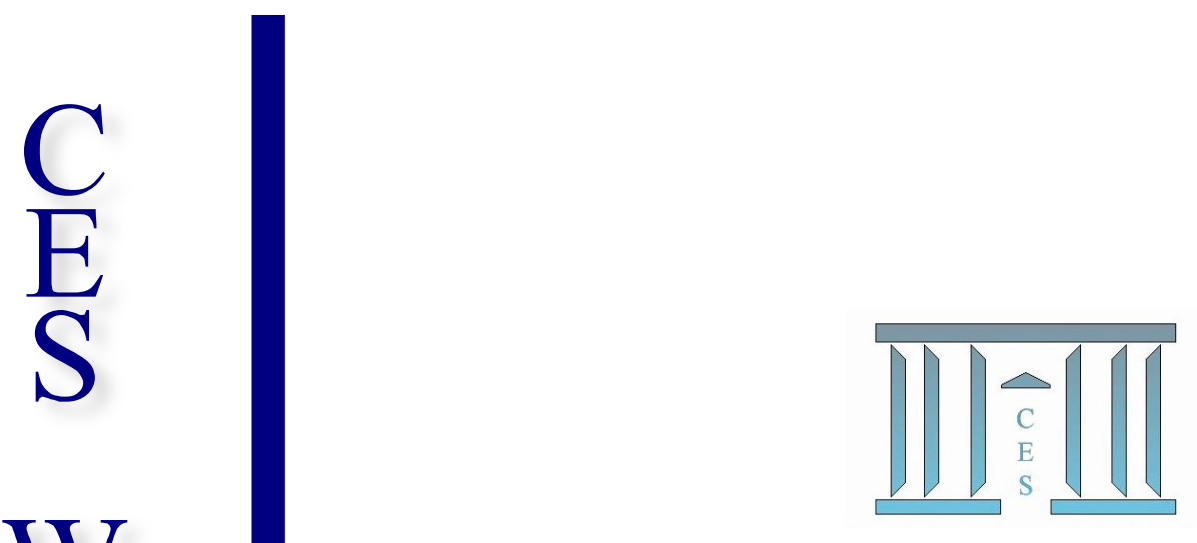

A Simple Probabilistic Approach of the Yard-Sale Model

Christophe CHORRO

$p$
$a$
$p$
$e$
p

2015.62

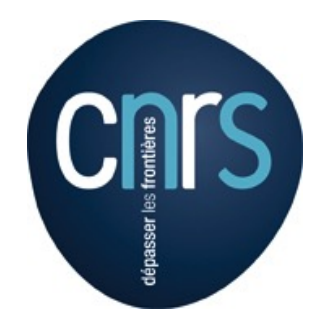




\title{
A Simple Probabilistic Approach of the Yard-Sale Model
}

\author{
Christophe Chorro*
}

Summer 2015

\begin{abstract}
We propose in the present paper a probabilistic approach of the so-called Hayes (2002) Yard-Sale model using classical diffusion approximations of Markov chains. We partly recover, at the very least for small and high frequency transactions, recent results of Boghosian (2014) and Boghosian et al. (2015) concerning both wealth condensation in the absence of redistribution mechanisms and steady state distributions when a uniform capital taxation is imposed.
\end{abstract}

Keywords: Wealth distribution, Yard-Sale model, Diffusion approximations, Impact of taxation.

\section{Introduction}

Several recent studies have questioned the impact of randomness on wealth concentration and proved that luck alone may generate extreme disparities in wealth dynamics even if economical agents are identical in terms of their patience and their abilities (see for example, Fernholz and Fernholz (2014), Boghosian et al. (2015) and Bouleau and Chorro (2015)). In these models, when there are no redistributive mechanisms, situations of strong concentration appear

${ }^{*}$ Corresponding author. Centre d'Economie de la Sorbonne, Maison des Sciences Economiques, 106 bd de l'Hopital, 75013 Paris, France. Email: cchorro@univ-paris1.fr. 
in which all the wealth ends up in the hands of a single oligarch and taxation is necessary to ensure the existence of a steady state distribution of wealth. Among these approaches, Multiplicative Random Asset Exchange Models (MRAEM), introduced in the framework of Econophysics by Ipsolatov et al. (1998), have recently emerged as interesting alternatives to proportional random growth models (see champernowne (1953) or Levy (2005)) to describe the evolution of wealth distribution in a population interacting economically. In MRAEM, economy is considered in its simplest form as an interchange of wealth between pairs of agents. This simplicity makes it possible to study social and collective phenomena. In particular, for the so-called Hayes (2002) Yard-Sale model, where a player can win with probability 0.5 a fraction of wealth of the poorer agent, Boghosian et al. (2015) show wealth concentration proving that the associated Gini (1921) coefficient, a classical measure of wealth inequality, is an $H$ function of the Boltzmann equation derived in Boghosian (2014). In the latter paper, the author also studies the impact of a uniform taxation of capital on wealth dynamics that leads to a steady state distribution for this economic system.

The aim of this paper is to recover these results, at the very least for small and high frequency transactions, using classical probabilistic diffusion approximations in the spirit of Chorro and Bouleau (2015). The main idea is to prove the convergence of the infinitesimal generator associated to the Yard-Sale repeated Markov chain game by transforming the time scales and state spaces appropriately and to study the limiting diffusion process.

For simplicity, we start, in Section 2, with the case of two players where the computations are explicit. The extension to the $N$ players game is discussed in Section 3. Section 4 provides some conclusions.. 


\section{The two players case}

In the so-called Yard-Sale model, at each round, each player may win or lose, with the same probability, a fraction $a$ of the wealth owned by the poorest agent ${ }^{1}$. This model has been introduced in Hayes (2002) to overcome some economic bias of the Theft-and-Fraud model of Ipsolatov et al. (1998) where the exchanged amount is a fraction of the losing agent's wealth. In fact, the last model drastically favors the poorest player while the Yard-Sale one is fair in expectation. More precisely, in the Yard-Sale model, if we denote by $X_{n}^{i}$ the wealth of player $i \in\{1,2\}$ after $n$ transactions, supposing that ${ }^{2} X_{0}^{1}+X_{0}^{2}=1$, we have $X_{n}^{1}+X_{n}^{2}=1$ (zero-sum game) and

$$
X_{n+1}^{i}=X_{n}^{i}+a \min \left(X_{n}^{i}, 1-X_{n}^{i}\right) \mathbf{1}_{U_{n+1} \leqslant \frac{1}{2}}-a \min \left(X_{n}^{i}, 1-X_{n}^{i}\right) \mathbf{1}_{U_{n+1}>\frac{1}{2}}
$$

where $\left(U_{k}\right)_{k \in \mathbb{N}^{*}}$ is a sample of the uniform distribution on $[0,1]$. The sequence $\left(X_{n}^{i}\right)_{n \in \mathbb{N}}$ is a Markov chain with $\mathbb{E}\left[X_{n+1}^{i} \mid X_{n}^{i}\right]=X_{n}^{i}$. Thus $\left(X_{n}^{i}\right)_{n \in \mathbb{N}}$ is a non-negative and bounded martingale that converges almost-surely and in $L^{p}(1 \leqslant p<\infty)$ toward a random variable $X_{\infty}^{i}$. From

$$
\mathbb{E}\left[\left(X_{n+1}^{i}-X_{n}^{i}\right)^{2} \mid X_{n}^{i}\right]=a^{2} \min \left(X_{n}^{i}, 1-X_{n}^{i}\right)^{2}
$$

we deduce that $X_{\infty}^{i}$ follows a Bernoulli distribution of parameter $X_{0}^{i}$. Thus, even if it is not possible for one player to be ruined after a finite number of rounds, all the wealth is concentrated at the limit in the hands of a single player.

By transforming the time scales and state spaces appropriately it is possible to study the continuous time limit of the preceding Markov chain game. This methodology was used in Bouleau and Chorro (2015) to obtain theoretical approximations of almost-bankruptcy times

\footnotetext{
${ }^{1}$ The parameter $1-a$ a may be seen as a measure of the saving propensity supposed to be constant among all the participants to ensure an identical involvement.

${ }^{2}$ The zero-sum game hypothesis implies that no wealth is imported, exported, generated, or consumed, wealth can only change hands. Working in a closed economic system, we use in this paper proportions of wealth instead of absolute values.
} 
in elementary market games with proportional bets: Let $f: \mathbb{R} \rightarrow \mathbb{R}$ be a measurable and bounded mapping. For all $a \in \mathbb{R}_{+}$and $\left.x \in\right] 0,1$ [ we define the generator $A_{a}$ of the elementary market game with parameter $a$ :

$A_{a}[f](x)=\mathbb{E}\left[f\left(X_{1}^{1}\right)-f\left(X_{0}^{1}\right) \mid X_{0}^{1}=x\right]=\frac{1}{2} f(x+a \min (x, 1-x))+\frac{1}{2} f(x-a \min (x, 1-x))-f(x)$.

In particular, when $f$ is of class $C^{\infty}$ with a compact support in the interval $] 0,1[$, we obtain from Taylor expansion that $\frac{1}{a^{2}} A_{a} f$ uniformly converges toward $\frac{1}{2} \min (x, 1-x)^{2} f^{\prime \prime}(x)$ when $a$ goes to 0 .

Considering the process $\left(Z_{t}^{a}\right)_{t \in \mathbb{R}_{+}}$that is the rescaled (at frequency $a^{2}$ ) continuous time linear interpolation of the sequence $\left(X_{n}^{1}\right)_{n \in \mathbb{N}}$ with $X_{0}^{1}=x$ :

$$
\begin{aligned}
Z_{n a^{2}}^{a} & =X_{n}^{1} \quad \forall n \geqslant 0 \\
Z_{(n+\theta) a^{2}}^{a} & =Z_{n a^{2}}^{a}+\theta\left(Z_{(n+1) a^{2}}^{a}-Z_{n a^{2}}^{a}\right) \quad \theta \in[0,1] \quad \forall n \geqslant 0,
\end{aligned}
$$

we obtain from classical arguments (see for example Stroock and Varadhan (1979), Chap. 11) the uniform weak convergence of $\left(Z_{t}^{a}\right)_{t \in \mathbb{R}_{+}}$when $a$ goes to 0 toward the diffusion process $\left(X_{t}\right)_{t \in \mathbb{R}_{+}}$, associated to the infinitesimal generator

$$
A[f](x)=\frac{1}{2} \min (x, 1-x)^{2} f^{\prime \prime}(x)
$$

that is the unique strong solution of the Stochastic differential equation

$$
d X_{t}=\min \left(X_{t}, 1-X_{t}\right) d B_{t} \quad 0<X_{0}<1
$$

where $B_{t}$ is a standard Brownian motion ${ }^{3}$. The points 0 and 1 are absorbing since the constant

\footnotetext{
${ }^{3}$ This result of convergence requires the existence and the unicity of the martingale problem associated to the generator (1) that is equivalent to the weak existence and the unicity in distribution of the solution of the associated stochastic differential equation. Here, (2) having Lipschitz we classically deduce the strong existence and unicity of the solution.
} 
processes 0 and 1 are solutions of $(2)$. The process $\left(X_{t}\right)_{t \in \mathbb{R}_{+}}$is then a continuous and uniformly integrable martingale that converges almost-surely toward 1 with probability $X_{0}$ and toward 0 with probability $\left(1-X_{0}\right)$. Nevertheless, since the Green's function (see Etheridge (2012), p.44) associated to the diffusion is given by $\forall x \in[0,1]$,

$$
\begin{aligned}
G(x, \xi) & =\frac{2 x(1-\xi)}{\min (\xi, 1-\xi)} \text { if } x<\xi<1 \\
& =\frac{2 \xi(1-x)}{\min (\xi, 1-\xi)} \text { if } 0<\xi<x
\end{aligned}
$$

we obtain from the Dynkin's formula that

$$
\mathbb{E}\left[T \mid X_{0}=x\right]=+\infty
$$

where $T$ is the hitting time of the boundary $\{0,1\}$ that is inaccessible in finite time. The economy never collapses in finite time in this case (contrary to what happens in Bouleau and Chorro (2015) for elementary market games with proportional bets), even if the concentration of wealth becomes stronger and stronger. In this case, this is not absorption because the absorption time is infinite with probability one, rather it is quasi-absorption ${ }^{4}$.

Now we study the impact of a small tax rate on the preceding dynamics to show that it is possible to affect the dispersion of the wealth previously observed. One of the simplest redistribution mechanism is to consider a proportional capital tax rate that is collected at any stage and uniformly reallocated to the players ${ }^{5}$. With a tax rate $b$ ( $b$ fulfilling $0<a+b<1$ ), the transition of the Markov chain becomes

$$
X_{n+1}^{i}=(1-b) X_{n}^{i}+a \min \left(X_{n}^{i}, 1-X_{n}^{i}\right) \mathbf{1}_{U_{n+1} \leqslant \frac{1}{2}}-a \min \left(X_{n}^{i}, 1-X_{n}^{i}\right) \mathbf{1}_{U_{n+1}>\frac{1}{2}}+\frac{b}{2}
$$

\footnotetext{
${ }^{4}$ If we denote by $T_{\varepsilon}$ the first exit time of the interval $[\varepsilon, 1-\varepsilon]$ we can prove using Etheridge (2012), p.44, that $\mathbb{E}\left[T_{\varepsilon} \mid X_{0}=x\right] \underset{0^{+}}{\sim}-2 \log (\varepsilon)$ quantifying how inaccessible the boundaries of this model are. This quantity is greater for small $\varepsilon$ than $-2[(1-x) \log (1-x)+x \log x]$, the limit that was obtained in Bouleau and Chorro (2015) for the Wright-Fisher diffusion without mutations associated to elementary market games with proportional bets.

${ }^{5}$ Is is easy to prove that if we consider instead a tax on the income a completely different asymptotic behavior is obtained. In this case, we recover (2) up to a constant: a proportional income tax is not sufficient to prevent agents from being ruined.
} 
where $\left(U_{k}\right)_{k \in \mathbb{N}^{*}}$ is a sample of the uniform distribution on the unit interval. In this case, the Markov chain is not a martingale anymore because

$$
\left.\mathbb{E}\left[X_{n+1}^{1}-X_{n}^{1}\right) \mid X_{n}^{1}\right]=b\left(\frac{1}{2}-X_{n}^{1}\right)
$$

The Yard-Sale model with capital taxation favors the poorest player during each transaction to mitigate the negative impact of randomness on wealth concentration. However, in spite of its simplicity, it is a priori difficult to obtain explicitly one invariant measure of such a Markov chain when $b \neq 0$. Therefore, we study directly the diffusion limit of the model for small and high-frequency transactions. Using the preceding argument the generator $A_{a}$ of the elementary taxed market game with parameters $a$ and $b$ becomes

$$
\begin{aligned}
A_{a}[f](x)=\mathbb{E}\left[f\left(X_{1}^{1}\right)-f\left(X_{0}^{1}\right) \mid X_{0}^{1}=x\right] & =\frac{1}{2} f\left((1-b) x+a \min (x, 1-x)+\frac{b}{2}\right) \\
& +\frac{1}{2} f\left((1-b) x-a \min (x, 1-x)+\frac{b}{2}\right)-f(x)
\end{aligned}
$$

in particular, when $f$ is of class $C^{\infty}$ with a compact support in the interval $] 0,1\left[, \frac{1}{a^{2}} A_{a} f\right.$ uniformly converges toward

$$
A[f](x)=\frac{1}{2} \min (x, 1-x)^{2} f^{\prime \prime}+\frac{\lambda}{2}(1-2 x) f^{\prime}
$$

when $a$ goes to 0 and $b=\lambda a^{2}{ }^{6}$ The generator $A$ is associated to the diffusion

$$
d X_{t}=\min \left(X_{t}, 1-X_{t}\right) d B_{t}+\frac{\lambda}{2}\left(1-2 X_{t}\right) d t \quad 0<X_{0}<1
$$

In this case, the scale function of the generator (3) is given by

$$
S(x)=\int_{1 / 2}^{x} \exp \left[-\int_{1 / 2}^{y} \frac{\lambda(1-2 z)}{\min (z, 1-z)^{2}} d z\right] d y,
$$

\footnotetext{
${ }^{6}$ Let us remark that in Boghosian (2014), the same hypothesis is made on the scale of the tax rate with respect to $a$ in order to obtain a Fokker-Planck equation for the steady state density of wealth when $a$ is small.
} 
thus,

$$
S^{\prime}(x)=K\left(e^{\lambda / x} x^{2 \lambda} \mathbf{1}_{x \leqslant \frac{1}{2}}+e^{\lambda /(1-x)}(1-x)^{2 \lambda} \mathbf{1}_{x>\frac{1}{2}}\right)
$$

In particular, $S(0+)=-\infty$ and $S(1-)=+\infty$ so the process is recurrent (see [13] Ex. 3.21 p. 298) and the associated invariant measure is given by the density (symmetric about 0.5$)^{7}$

$$
m_{2}(x)=C\left(e^{-\lambda / x} x^{-2(\lambda+1)} \mathbf{1}_{x \leqslant \frac{1}{2}}+e^{-\lambda /(1-x)}(1-x)^{-2(\lambda+1)} \mathbf{1}_{x>\frac{1}{2}}\right)
$$

where $C$ is a constant of integration. This density function is represented in Figure 1 for different values of $\lambda$. We remark that the mode of the invariant density on $[0,0.5]$ that is equal to $\frac{\lambda}{2 \lambda+2}$ converges toward 0.5 (egalitarian case) as $\lambda$ goes to infinity. For small $x$, we interestingly recover the expression provided, using the techniques of mathematical physics, in Boghosian (2014), Eq. 91 (with $W=1$ and $N=2$ ) with a different probabilistic approach based on diffusion approximations of Markov chains. In Figure 1 we also observe the impact of $\lambda$ on the wealth concentration process and we see that the proportion of wealth held by the wealthiest player falls drastically even for small values of the capital tax rate ${ }^{8}$.
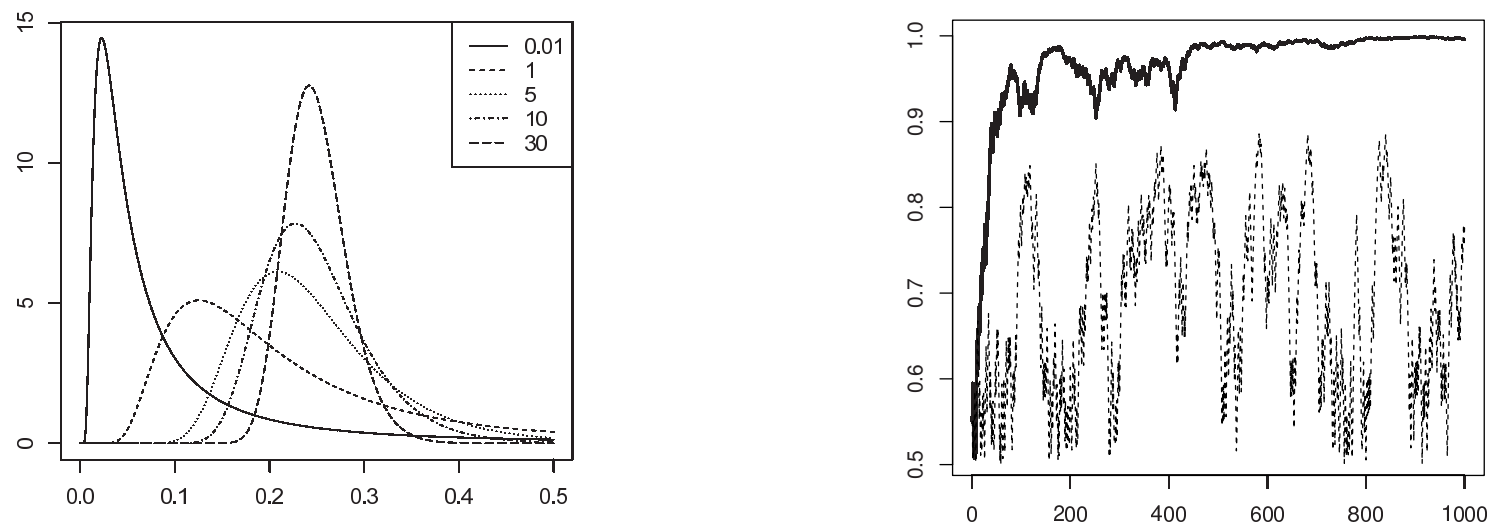

Figure 1: Steady state density (left) of the two players Yard-Sale model for different values of the capital tax rate $\lambda$ and proportion of the wealth (right) owned by the wealthiest player without (bold) or with (dashed) a $1 \%$ capital taxation in the two players game with $n=1000$ transactions. The invariant densities are represented on the interval $[0,0.5]$ because they are symmetric about 0.5 .

\footnotetext{
${ }^{7}$ In order to introduce asymmetry in the steady state density, we may consider a different tax rate for the two players.

${ }^{8}$ In simulations that are not reported here, we also observe that richest individuals tend not to remain the richest ones over time when the capital tax rate increases. In the Yard-Sale model social mobility and wealth concentration are highly correlated as already observed in Bouleau and Chorro (2015) and Fernholz (2015) for different models.
} 


\section{Remarks on the $N$ players case}

In a population of $N$ players, we select, during each round, two agents randomly and without replacement for a transaction according to the 2-player Yard-Sale mechanism described in the preceding section. We also consider in this framework a proportional capital taxation (associated to the rate $b$ ) uniformly distributed among all the players. The aim of this section is to present the main lines of the extension of the preceding results.

If we denote by $X_{n}=\left(X_{n}^{1}, \ldots, X_{n}^{N}\right)$ the vector of wealth after $n$ rounds and by $S_{N}$ the set $\left\{k=\left(k_{1}, k_{2}\right) \in \mathbb{N}^{2} \mid N \geqslant k_{1}>k_{2} \geqslant 1\right\}$, we have, $\forall n \in \mathbb{N}$,

$$
X_{n+1}=(1-b) X_{n}+\frac{b}{N}+\sum_{k \in S_{N}} Y_{n+1}^{k_{1}, k_{2}} \mathbf{1}_{V_{n+1}=\left(k_{1}, k_{2}\right)}
$$

with

$$
Y_{n+1}^{k_{1}, k_{2}}=a \min \left(X_{n}^{k_{1}}, X_{n}^{k_{2}}\right) \mathbf{1}_{U_{n+1}^{\left(k_{1}, k_{2}\right)} \leqslant \frac{1}{2}}\left(e_{k_{1}}-e_{k_{2}}\right)+a \min \left(X_{n}^{k_{1}}, X_{n}^{k_{2}}\right) \mathbf{1}_{U_{n+1}^{\left(k_{1}, k_{2}\right)}>\frac{1}{2}}\left(e_{k_{2}}-e_{k_{1}}\right)
$$

where $\left(V_{n}, U_{n}^{k}\right)_{(n, k) \in \mathbb{N}^{*} \times S_{N}}$ are independent random variables such that $V_{n}$ (resp. $\left.U_{n}^{k}\right)$ follows a uniform distribution on $S_{N}$ (resp. $[0,1]$ ) and where $e_{i}$ denotes the vector of $\mathbb{R}^{N}$ with a 1 in the i-th coordinate and 0's elsewhere. Starting from a point $X_{0}$ in the simplex $\left\{x=\left(x_{1}, \ldots, x_{N}\right) \in \mathbb{R}^{N} \mid \sum_{i=1}^{N} x_{i}=1, x_{i} \geqslant 0\right\},\left(X_{n}\right)_{n \in \mathbb{N}}$ stays in the simplex and for $b=0$, $\left(X_{n}\right)_{n \in \mathbb{N}}$ is once again a bounded martingale that converges almost surely and in $L^{p} 1 \leqslant p<$ $+\infty$, toward $X_{\infty}$ that is invariant with respect to the transition of the chain. From

$$
\mathbb{E}\left[\left|X_{n+1}-X_{n}\right|^{2} \mid X_{n}\right]=\sum_{k=\left(k_{1}, k_{2}\right) \in S_{N}} N(N-1) a^{2} \min \left(X_{n}^{k_{1}}, X_{n}^{k_{2}}\right)^{2}
$$

we deduce that $X_{\infty}$ almost surely belongs to the vertices of the simplex and that $X_{\infty}=e_{i}$ with probability $X_{0}^{i}$. This strong tendency for the Yard-Sale model to drive increasing fractions of wealth into the hands of a single agent has been already observed numerically in Sinha 
(2003) and Boghosian (2014) and we provide in this paper a very simple probabilistic proof of this phenomenon ${ }^{9}$. Now, supposing that $b=\lambda a^{2}>0$, we can prove, along the same lines as the preceding section, that the generator associated to the $N$ players game with proportional capital taxation is equal, for $F: \mathbb{R}^{N} \rightarrow \mathbb{R}$ of class $C^{\infty}$ with compact support, to

$$
\begin{aligned}
A_{a}[F](x)=\frac{1}{N(N-1)} \sum_{k=\left(k_{1}, k_{2}\right) \in S_{N}}[ & {\left[F\left(x+a \min \left(x_{k_{1}}, x_{k_{2}}\right)\left(e_{k_{1}}-e_{k_{2}}\right)\right)-F(x)\right.} \\
& \left.F\left(x+a \min \left(x_{k_{1}}, x_{k_{2}}\right)\left(e_{k_{2}}-e_{k_{1}}\right)\right)-F(x)\right]
\end{aligned}
$$

and that the rescaled continuous time linear interpolation at frequency $a^{2}$ of the sequence $\left(X_{n}\right)_{n \in \mathbb{N}}$ converges in distribution towards the diffusion associated to the infinitesimal generator

$$
\begin{aligned}
A_{\lambda}^{N}[F](x) & =\frac{1}{N(N-1)} \sum_{k=\left(k_{1}, k_{2}\right) \in S_{N}} \min \left(x_{k_{1}}, x_{k_{2}}\right)^{2}\left(F_{k_{1} k_{1}}^{\prime \prime}(x)+F_{k_{2} k_{2}}^{\prime \prime}(x)-2 F_{k_{1} k_{2}}^{\prime \prime}(x)\right) \\
& +\sum_{i=1}^{N} \frac{\lambda}{N}\left(1-N x_{i}\right) F_{i}^{\prime}(x)
\end{aligned}
$$

Of course we recover for $N=2$ the expression of Equation (3), however, up to our knowledge, there exists no classical expression for the steady state distribution associated to this generator for $N>2$. Nevertheless, it can be proved easily that the steady state one-dimensional marginal distributions of this model are obtained, for small $x$, from the one dimensional generator

$$
\frac{x^{2}}{N} f^{\prime \prime}+\frac{\lambda}{N}(1-N x) f^{\prime}
$$

Thus, for small $x$, the steady state one-dimensional marginal distributions take the form

$$
m_{N}(x)=C_{N} e^{-\lambda / x} x^{-N \lambda-2}
$$

(where $C_{N}$ is a constant of integration) with a mode that is equal to $\frac{\lambda}{N \lambda+2}$. This expression

\footnotetext{
${ }^{9}$ In Boghosian et al. (2015), the authors prove this result demonstrating that the Gini (1921) coefficient is a Lyapunov function of the Boltzmann equation for the Yard-Sale model. Thus this coefficient increases in time and asymptotically approaches the state of perfect inequality.
} 
is similar ${ }^{10}$ to Boghosian (2014), Eq. 91 obtained by the author using a random-agent approximation that is classical in Econophysics but different from our approach where diffusion approximations of Markov chains are simply used. In Figure 2, we have represented these theoretical approximations for the games with $N=10$ and $N=50$ players (with $\lambda=1 \%$ ) to compare them with the one-dimensional empirical marginals obtained from the components of the Markov chain dynamics by Monte Carlo simulations: the results are close together for small values of $x$.
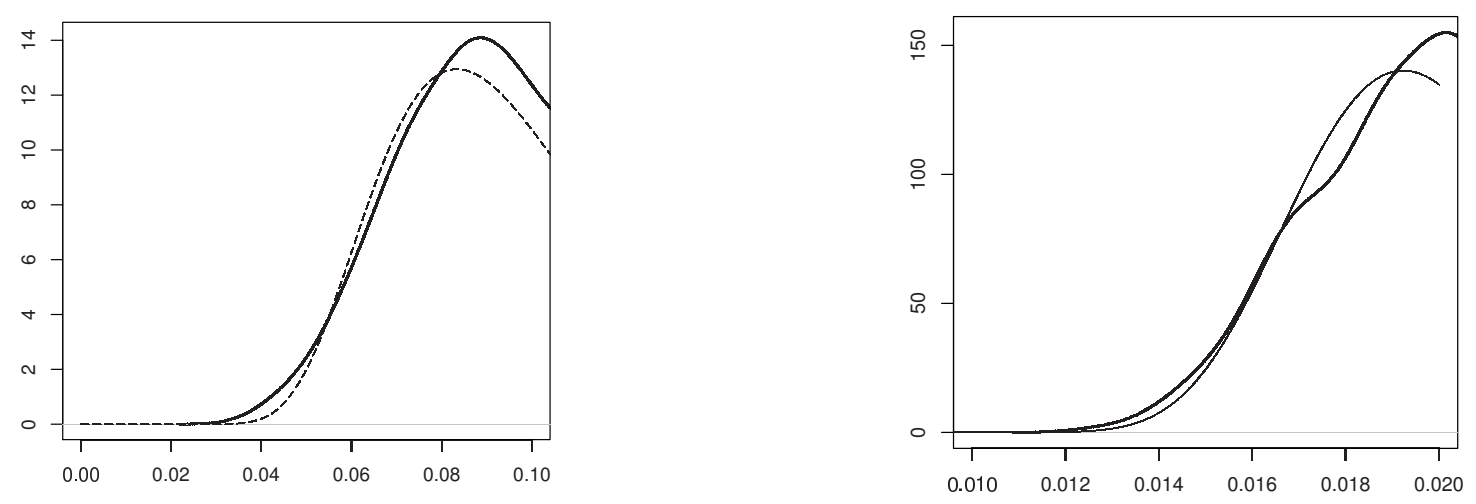

Figure 2: Invariant density function of the Yard-Sale model with proportional capital taxation $(\lambda=$ $1 \%$ ) for $N=10$ (left part) and $N=50$ (right part). We represent, for small values of $x$, the empirical density function (bold) obtained using 1000 independent Monte Carlo simulations of $X_{100000}^{1}$ in the Markov chain market game with parameters $a=0.1$ and $b=a^{2}$ and the density obtained from the diffusion approximation.

Remark: Another possible extension of the two players game is to consider that all the agents play simultaneously and that during each round one player can win a proportion $a$ of $\min \left(X_{n}^{i}\right)$ (divided equally between the other players) with probability $\frac{1}{N}$. In its taxed version, this Markov chain game may be written in the following way:

$$
X_{n+1}=(1-b) X_{n}-\frac{a}{N-1} \min \left(X_{n}^{i}\right)+\frac{a N}{N-1} \min \left(X_{n}^{i}\right) e_{i}+\frac{b}{N} \text { with probability } \frac{1}{N} .
$$

Supposing once again that $b=\lambda a^{2}>0$, we can prove that the associated generator converges

\footnotetext{
${ }^{10}$ Such a form for the steady state distribution is also obtained for wealth distribution problems in a slight different model by Bouchaud and Mezard (2000).
} 
toward

$$
\tilde{A}_{\lambda}^{N}[F](x)=\frac{\min \left(x_{i}\right)^{2}}{2(N-1)^{2}} \sum_{i, j=1}^{N}\left(N \delta_{i j}-1\right) F_{i j}^{\prime \prime}(x)+\sum_{i=1}^{N} \frac{\lambda}{N}\left(1-N x_{i}\right) F_{i}^{\prime}(x)
$$

where $\delta_{i j}=1$ if $i=j$ and 0 otherwise. For $N=2$ we recover the Equation (3) and for $N>2$ this generator is different from $A_{\lambda}^{N}$.

\section{Conclusion}

This paper provides a simple alternative probabilistic study of the Yard-Sale model. First, we give an elementary proof of the concentration of wealth, when there is no redistribution, with a different approach than Boghosian et al. (2015). Then, using classical approximations of Markov chains by continuous diffusions, we study the steady state probability of the system when proportional capital taxation is added to the initial dynamics. In particular, we recover for small and high frequency transactions, the approximation, in the vicinity of 0 , of the one-dimensional steady state marginals obtained in Boghosian (2014) with an Econophysics view.

\section{References}

[1] Boghosian, B.M., (2014). Kinetics of Wealth and the Pareto Law. Physical Review E, 89(4), 042804-042825.

[2] Boghosian, B.M., Jonhson, M. and Marcq, J.A., (2015). An H theorem for Boltzmann's equation for the Yard-Sale Model of asset exchange (The Gini coefficient as an H functional). To appear in Journal of Statistical Physics.

[3] Bouchaud, J.P. and Mezard, M., (2000). Wealth Condensation in a Simple Model of Economy. Physica A, 282, 536-545. 
[4] Bouleau, N. and Chorro, C., (2015). The impact of randomness on the distribution of wealth: Some economic aspects of the Wright-Fisher diffusion process. Cahiers du CES.

[5] Champernowne, D.G., (1953). A Model of Income Distribution. Economic Journal, 63, 318-351.

[6] Etheridge, A., (2012). Some Mathematical Models from Population Genetics. École d'Été de Probabilités de Saint-Flour XXXIX-2009. Lecture notes in Mathematics. Springer.

[7] Fernholz, R.T. and Fernholz, R., (2014). Instability and concentration in the distribution of wealth. Journal of Economic Dynamics and Control, 44, 251-269.

[8] Fernholz, R.T., (2015). A model of economic mobility and the distribution of wealth. Mimeo, Claremont McKenna College.

[9] Gini, C., (1921). Measurement of inequality of income. Economic Journal, 31, 22-43.

[10] Hayes, B., (2002). Follow the Money. American Scientist, 90, 400-405.

[11] Ispolatov, C., Krapivsky, P.L. and Redner, S., (1998). Wealth distributions in asset exchange models. The European Physical Journal B - Condensed Matter and Complex Systems, 2(2), 267-276.

[12] Levy, M., (2005). Market efficiency, the Pareto wealth distribution, and the Levy distribution of stock returns. In The Economy As an Evolving Complex System, III: Current Perspectives and Future Directions. Oxford University Press.

[13] Revuz, D. and Yor, M., (1994). Continuous Martingales and Brownian Motion. Springer.

[14] Sinha, S., (2003). Stochastic Maps, Wealth Distribution in Random Asset Exchange Models and the Marginal Utility of Relative Wealth. Physica Scripta, 106, 59-64.

[15] Stroock, D.W. and Varadhan, S.R.S., (1979). Multidimensional diffusion processes. Springer. 